\title{
Malnutrition Risk, Physical Function Decline and Disability in Middle-Aged and Older Adults Followed Up in the Singapore Longitudinal Ageing
} Study

\author{
Shan $\mathrm{Hai}^{1}$ \\ Qi Gao ${ }^{2}$ \\ Xinyi Gwee ${ }^{2}$ \\ Denise Qian Ling Chua ${ }^{2}$ \\ Keng Bee Yap ${ }^{3}$ \\ Tze Pin Ng $\mathbb{D}^{2}$ \\ 'Gerontology and Geriatrics Centre, \\ West China Hospital of Sichuan \\ University, Chengdu, People's Republic of \\ China; ${ }^{2}$ Department of Psychological \\ Medicine, Yong Loo Lin School of \\ Medicine, National University of \\ Singapore, Singapore; ${ }^{3}$ Department of \\ Medicine, Ng Teng Fong General \\ Hospital, Singapore
}

Correspondence: Tze Pin Ng Department of Psychological Medicine, Yong Loo Lin School of Medicine, National University of Singapore, Level 9, NUHS Tower Block, IE Kent Ridge Road, II 9228 , Singapore

Tel $+6567724518 ;+6567723478$

Fax +6567772191

Email pcmngtp@nus.edu.sg
Purpose: Malnutrition and population ageing are doubly global healthcare challenges. It is widely recognized that disability is a major contributor to malnutrition among older people, but the importance of reducing malnutrition risk (MR) for disability prevention is given little attention. We investigated the association between MR and risk of incident disability and functional decline among community-dwelling older persons.

Materials and Methods: Prospective cohort study of community-dwelling older adults aged 55 and above (Singapore Longitudinal Ageing Study) with 3-5 years follow-up. MR was measured at baseline using an appropriate and validated index (ENIGMA); physical and functional measures at baseline and follow-up included instrumental and basic activity of daily living (ADL), knee extension (KES), gait velocity (GV), timed up-and-go (TUG).

Results: Compared to low MR, mild-moderate MR (OR $=1.43,95 \% \mathrm{CI}=1.02-2.01)$ and severe MR $(\mathrm{OR}=1.74,95 \% \mathrm{CI}=1.10-2.74)$ were associated with higher risks of incident disability. Severe MR was associated with functional decline $(\mathrm{OR}=1.69,95 \% \mathrm{CI}=1.11-$ 2.57). Estimates were adjusted for demographic, social, lifestyle behaviour and health variables. In particular, eating difficulty was associated with incident disability and functional decline, and low lymphocyte with functional decline. Severe MR and low albumin were associated with $37 \%$ worse GV and $126 \%$ worse TUG declines; consuming few vegetables or fruits with $34 \%$ worse KES decline; polypharmacy with $56 \%$ worse TUG decline.

Conclusion: Malnutrition risk increased the risk of incident disability and functional decline in non-disabled persons, it worsened physical performance declines. Further studies should investigate the effectiveness of nutritional interventions in reducing the risk of disability among older people.

Keywords: malnutrition, functionality, ageing, prospective cohort study

\section{Introduction}

Malnutrition including undernutrition, micronutrient-related malnutrition, overweight, and obesity is a global problem with serious developmental, economic, social, and healthcare impacts. ${ }^{1}$ Malnutrition is especially common among older people. As many as a third of older adults in the community are at risk of malnutrition or malnourished ${ }^{2,3}$ for reasons particularly related to physiological (anorexia of ageing, impaired oral and gastrointestinal digestion, absorption and metabolism) and social factors (poverty, social isolation) in old age. ${ }^{4}$ Population ageing is a global challenge 
and is most critical in low- and middle-income countries. ${ }^{5}$ The health, social and economic impact of global population ageing are well documented. In particular, functional disability in old age is the primary determinant of the burden of long-term care and increased healthcare expenditure. ${ }^{6}$

The importance of recognizing and treating malnutrition among older people is widely recognised. ${ }^{7}$ Based on numerous studies of disabled, hospitalized or institutionalized older persons, it is widely recognized that functional disability contributes in a major way to the onset and worsening of malnutrition among older people. ${ }^{7}$ However, the influence of malnutrition risk in the disablement process leading eventually to functional disability, and the potential impact of nutritional interventions for preventing disability and thereby reducing long-term care burden are generally given much less attention. Studies that have investigated malnutrition risk as a risk factor for physical function decline and disability are mostly crosssectional, ${ }^{8-27}$ and longitudinally designed studies using appropriate measures of nutrition are few. ${ }^{22,23,26}$

In this study, we investigated the link between malnutrition risk and the risks of incident functional disability and decline in a prospective cohort study of communitydwelling older adults from 3 to 5 years of follow-up. We also examined in detail the associations between components of malnutrition risk and disability/functional decline. To explicate the mechanistic links in the disablement process, we examined among non-disabled individuals the associations between malnutrition risk and decline in muscle strength and functional mobility which are known precursors of disability. To minimize measurement bias from the circular relationship of malnutrition and functional disability, we used a recently validated index of malnutrition risk which has demonstrated high predictive validity for mortality risk ${ }^{28}$ and includes domain indicators of eating difficulty (chewing and swallowing), consuming few vegetables or fruits, taking 5 or more drugs, low total cholesterol, low albumin, low haemoglobin, low lymphocyte counts.

\section{Materials and Methods}

\section{Study Participants}

The Singapore Longitudinal Ageing Study (SLAS) is a population-based prospective cohort study of ageing and health transition among older adults, aged 55 and above in Singapore. ${ }^{28}$ Two population cohorts were studied in separate waves of recruitment in different geographical locations: SLAS-1 cohort comprised participants
$(\mathrm{N}=2804)$ who were recruited in 2003-2004 and were followed up at two re-assessment visits approximately 34 years apart in 2005-2007 and 2007-2009 respectively. SLAS-2 participants $(\mathrm{N}=3270)$ were recruited in 20092013, with follow-up assessments 3 to 5 (mean 4.5) years apart completed in 2013-2018.

Details of the study methodology and measurements have been previously reported. ${ }^{29,30}$ Briefly, study participants aged 55 and above were recruited in whole population sampling of residents aged 55 and above who were residents in contiguous neighbourhoods in the South East (SLAS1) and South-and-Western regions of the island. Participants with severe physical and/or cognitive disabilities or terminal illnesses who were unable to participate in questionnaire interviews or physical and cognitive performance testing were excluded. The extensive range of baseline personal and health data collected by trained research nurses and psychology research assistants included questionnaire, anthropometric and laboratory blood measurements related to food intake, nutritional risk and malnutrition. The study was conducted according to the guidelines laid down in the Declaration of Helsinki and all procedures involving human subjects were approved by the Institutional Review Board of National University of Singapore (NUS-IRB 04-140). Written informed consent was obtained from all subjects.

The present study involved a total of 5358 participants in the combined SLAS-1 and SLAS-2 cohort with available baseline data of nutritional and disability status who were assessed at the first follow-up on incident disability and ADL decline. Among disability-free participants in the SLAS-2 cohort, we analysed the association between malnutrition risk and physical performance using measures of knee extension strength (KES), gait velocity (GV) and timed-up-and-go (TUG) that were available at baseline and follow-up. See Flow Chart (Figure 1).

\section{Baseline Nutritional Status}

At baseline, nutritional risk information was obtained from items in the Nutritional Screening Initiative (NSI) questionnaire, $^{31}$ and the Mini Nutritional Assessment short form (MNA-SF), ${ }^{32}$ and serum levels of known blood biomarkers related to undernutrition, low immunity and mortality: low hemoglobin or anemia, ${ }^{33,34}$ low lymphocyte counts, ${ }^{27,35}$ low total cholesterol, ${ }^{36-38}$ and low albumin. ${ }^{24-27,36-38}$ For the purpose of this study, we used a composite index of malnutrition risk (Elderly Nutritional Indicators for Geriatric Malnutrition Assessment or ENIGMA) derived from these measurements. The 


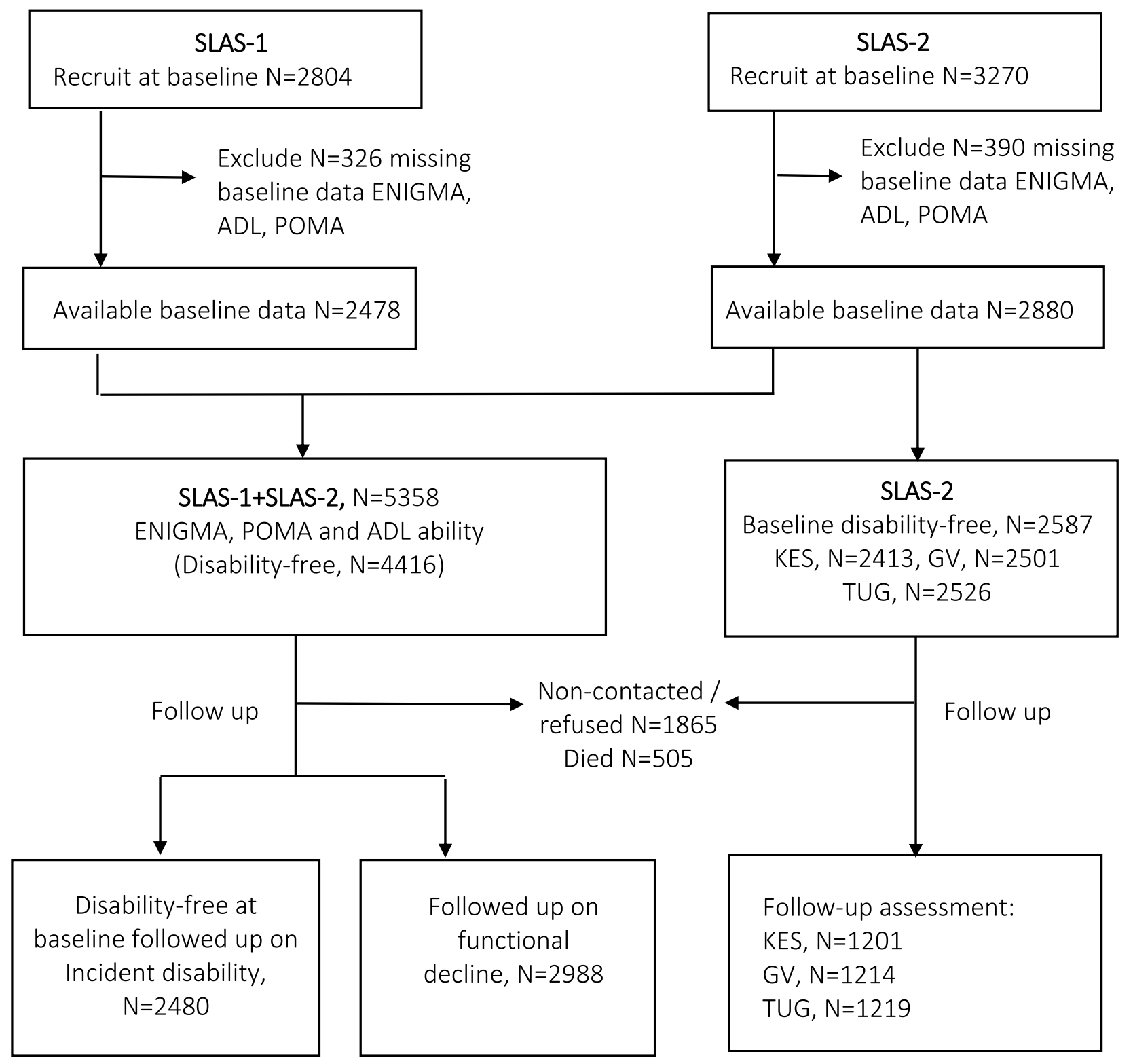

Figure I Flow chart of study participants recruitment and assessment.

ENIGMA malnutrition risk index included eight nutritional indicators (unable to shop, cook or feed one's self, eating difficulty due to oral problems, eat few vegetables or fruits (less than once a day)), taking 5 or more drugs a day, low albumin, haemoglobin, total cholesterol and lymphocyte count). The ENIGMA index was previously shown to have higher predictive accuracy for mortality than MNA, GNRI or underweight-by-ESPEN. ${ }^{23}$ Low body mass index (BMI) in the presence of other indicators was not predictive of mortality outcome and hence not included in the index. To minimize measurement bias, we excluded "unable to shop, cook or feed one's self" from the index in this study. The summed weighted scores for the seven nutritional indicators $(0=$ not present, $1=$ present for all indicators except "low cholesterol" which was given a score of 2 ), with potential scores from 0 to 10 were used to define three ordinal levels of malnutrition risk: $0=$ none, $1-2=$ low to moderate, $\geq 3=$ severe.

\section{Outcomes}

\section{Functional Disability}

Functional dependence (and physical and mobility performance) were assessed at baseline and follow-up independently by different research nurses. In the total cohort, disability was assessed by items on need for help in performing instrumental and basic activities of daily living 
(ADL) found in the Barthel and the Lawton scales, which have been previously validated in the Singaporean population. ${ }^{39,40}$ We used an expanded IADL-ADL scale measuring functional dependence as it has been shown to be hierarchical, unidimensional, and unbiased by age, with greater content validity and thus more useful than basic ADL for measuring need for help in the community. ${ }^{41,42}$ The presence of at least one ADL limitation was used to define prevalent disability at baseline, and incident disability at follow-up of participants who were disability-free at baseline. Functional decline in ADL was defined by an increase of at least one ADL limitation between baseline and follow-up for participants.

\section{Physical Performance}

In the SLAS-2 cohort, physical performance was assessed by knee extension strength (KES), gait velocity (GV) and timed-up-and-go (TUG).

Knee extension strength (KES) was measured for the lower limb maximum isometric strength. It was measured with the participant seated, the hip and knee angles at $90^{\circ}$ using the strap and strain gauge component of the Physiological Profile Assessment, ${ }^{43}$ using three trials' dominant leg average value (in kilograms).

Gait velocity $(\mathrm{GV})$ was measured by the time in seconds taken for the participant to walk 6 metres at their fastest pace, averaged for two trials. Participants performed the test with a dynamic start on a smooth, flat 10-metre walkway with redtape markers placed at the 0-, 2-, 8, and 10-metre points along the walkway, allowing for acceleration the first 2 metres and deceleration over the last 2 metres. The timing in a stopwatch is started when the toes of the leading foot cross the 2-meter mark and stopped when the toes of the leading foot cross the 8-meter mark.

Timed Up-and-Go (TUG) was measured by the time taken by the participant to stand up from an armchair (46 cm height), walk 3 metres, turn, walk back to the chair, and sit down again. The participants wore their regular footwear and used their customary walking aid, if required. Participants walked at their fastest pace with no physical assistance given. The test was administered twice, and the best performance time was used. ${ }^{44}$

\section{Covariates}

These included baseline socio-economic status: age, sex, ethnicity (Chinese, Malay and Indians/Others), educational level ( $\leq 6$ years, $7-10$ years, $\geq 11$ years), housing type (1-2-room public housing, 3-room public housing, $\geq 4$-room public housing and private housing); lifestyle and behaviour variables: single/widowed/divorced versus married status, living alone, physical activity (summed score of frequency of engagement in 4 categories of moderate to vigorous physical activities). Multi-morbidity (5 or more chronic diseases) was determined by the total number of chronic diseases in individual participants, derived from self-reports, and names of drugs from medication packages, or history of diagnostic or surgical procedures, for 18 listed medical diagnoses. The determination of hypertension included additionally measured systolic blood pressure $>130 \mathrm{mmHg}$ or diastolic blood pressure $>85 \mathrm{mmHg}$ among those with no history of hypertension diagnosis and treatment, and diabetes included additional fasting blood glucose $>5.6 \mathrm{mmol} / \mathrm{L}$ (pre-diabetes). Global cognition was assessed by locally translated version of the Mini Mental State Examination (MMSE), ${ }^{45}$ and depression by the Geriatric Depression Scale-15 items (GDS-15). ${ }^{46}$

\section{Statistical Analysis}

Data analysis was performed using SPSS statistical package (IBM version 25). We used analysis of variance for continuous data and chi-squared analyses for categorical data for comparisons of nutritional status groups. In the total cohort, the association of baseline ordinal MR score or component nutritional indicators with cumulative risks of incident disability and functional decline were analysed using logistic regression to estimate odds ratio (OR) and 95\% confidence intervals $(95 \% \mathrm{CI})$, controlling for socio-demographic, lifestyle behaviour and health variables. In the SLAS-2 cohort, and among baseline disability-free participants, the association between baseline MR with the levels and changes in physical performance measures were analysed using generalized linear mixed models for repeated measures. Estimates of levels and changes in KES, GV and TUG associated with nutritional status groups with significance testing of the effects of group, time and group by time interaction were adjusted for baseline covariates (above) and baseline KES, GV or TUG as appropriate.

\section{Results}

The mean age of the whole cohort was 66.6 (SD 7.6) years, and 63\% were women (Table 1). Low-moderate and severe MR was present in $47 \%$ and $15 \%$ of the participants respectively, attributed mostly to low albumin (25\%), low haemoglobin (17.3\%), and multiple drug use (15\%). The baseline prevalence of disability was $16.7 \%$, 
Table I Baseline Characteristics of Study Participants in Total (Combined SLAS-I and SLAS-2) Cohort and SLAS-2 Cohort

\begin{tabular}{|c|c|c|c|c|c|c|}
\hline & \multicolumn{3}{|c|}{ Total Cohort } & \multicolumn{3}{|c|}{ SLAS-2 Cohort } \\
\hline & Total & $\mathbf{N}$ & $\%$ & Total & $\mathbf{N}$ & $\%$ \\
\hline & $(N=5358)$ & Mean & \pm SD & $\mathbf{N}=\mathbf{2 8 8 0}$ & Mean & \pm SD \\
\hline Sex: female & & 3377 & 63.0 & & 1813 & 63.0 \\
\hline Age, years & & 66.6 & \pm 7.6 & & 66.3 & \pm 7.7 \\
\hline Ethnicity: Chinese & & 5006 & 93.4 & & 2528 & 87.8 \\
\hline Malay & & 212 & 4.0 & & 212 & 7.4 \\
\hline Indian & & 140 & 2.6 & & 140 & 4.9 \\
\hline Education: $\leq 6$ years & & 1857 & 34.7 & & 564 & 19.6 \\
\hline $7-10$ years & & 2001 & 37.3 & & 1218 & 42.3 \\
\hline$\geq I I$ years & & 1500 & 28.0 & & 1098 & 38.1 \\
\hline Housing: I-2 room public & & 764 & 14.3 & & 610 & 21.2 \\
\hline 3 room public & & 1404 & 26.3 & & 817 & 28.4 \\
\hline$\geq 4$ room and private & & 3190 & 59.6 & & 1453 & 50.4 \\
\hline Single/divorced/widowed & & 1625 & 30.3 & & 996 & 34.6 \\
\hline Live alone & & 625 & 11.7 & & 450 & 15.6 \\
\hline Physical activity score & & 4.5 & \pm 2.5 & & 6.2 & 1.5 \\
\hline$\geq 5$ medical illnesses & & 706 & 13.2 & & 523 & 18.2 \\
\hline MMSE score & & 27.4 & \pm 3.2 & & 27.8 & 2.8 \\
\hline GDS score & & 1.27 & \pm 2.2 & & 0.73 & $\mathrm{I} .47$ \\
\hline ENIGMA7 score & & 1.17 & $\pm \mid .31$ & & 1.30 & \pm 1.43 \\
\hline 0 (no MR) & & 2035 & 38.0 & & 1092 & 37.9 \\
\hline I-2 (low-medium MR) & & 2525 & 47.1 & & 1254 & 43.5 \\
\hline 3+ (high MR) & & 798 & 14.9 & & 534 & 18.5 \\
\hline Eating difficulty (chewing and swallowing) & & 353 & 6.6 & & 225 & 7.8 \\
\hline Take 5 or more drugs & & 808 & 15.1 & & 544 & 18.9 \\
\hline Few fruits or vegetables & & 498 & 9.3 & & 295 & 10.2 \\
\hline Low total cholesterol & & 609 & 11.4 & & 418 & 14.5 \\
\hline Low albumin & & $134 \mid$ & 25.0 & & 614 & 21.3 \\
\hline Low haemoglobin & & 927 & 17.3 & & 588 & 20.4 \\
\hline Low lymphocyte counts & & 351 & 6.6 & & 204 & 7.1 \\
\hline Baseline prevalent IADL-BADL disability & & 897 & 16.7 & & 293 & 10.2 \\
\hline
\end{tabular}

Abbreviation: NA, not analysed (data not available in SLAS-I cohort).

increasing from $11.6 \%$ to $18.2 \%$ and $25.2 \%$ across ordinal categories of poor nutrition (Table 2).

\section{Incident Disability and Functional Decline}

Among participants who were free of disability at baseline, the cumulative incidence of disability was $8.9 \%$ overall, and increased from $5.9 \%$ to $10.1 \%$ and $14.9 \%$ across the ordinal categories of MR (Table 2 and Supplementary Figure 1). The OR of association adjusted for demographic, social, lifestyle behaviour and health variables for low-moderate $\mathrm{MR}$ was $1.43(95 \% \mathrm{CI}=1.02$, 2.01), and for severe MR was 1.74 (95\% CI=1.10, 2.74). 


\begin{tabular}{|c|c|c|c|c|c|c|c|c|c|c|}
\hline \multirow{5}{*}{ 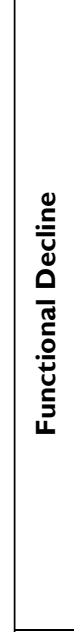 } & 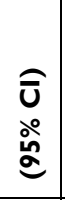 & & 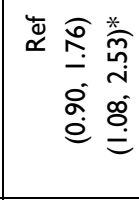 & 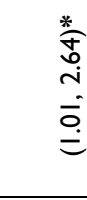 & 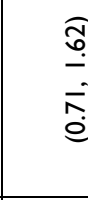 & 每 & $\begin{array}{l}\frac{6}{6} \\
\stackrel{e}{e} \\
\stackrel{e}{e}\end{array}$ & 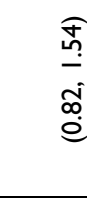 & $\begin{array}{l}\stackrel{\widehat{0}}{\stackrel{0}{0}} \\
\stackrel{\vec{\infty}}{\stackrel{0}{e}}\end{array}$ & 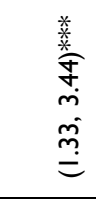 \\
\hline & $\stackrel{\circ}{0}$ & & 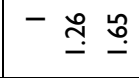 & 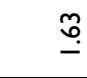 & $\stackrel{i}{-}$ & $\stackrel{\infty}{\sigma}$ & $\bar{E}$ & $\stackrel{\cong}{=}$ & $\stackrel{\circ}{=}$ & $\stackrel{ \pm}{i}$ \\
\hline & $\mathbf{\mathbf { z }}$ & 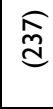 & 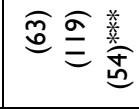 & 尊善 & 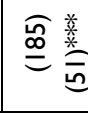 & $\widehat{\overline{\mathrm{a}}} \overline{\mathrm{d}}$ & 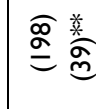 & 喬善 & 鸽䊦 & $\frac{\widehat{o}}{\mathrm{~d}} \frac{\mathrm{i}}{\mathrm{d}}$ \\
\hline & $\circ$ & $\stackrel{\leftrightarrow}{\sim}$ & 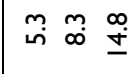 & $\stackrel{\star}{\sim} \stackrel{0}{0}$ & $\bar{n} \stackrel{\underline{n}}{\underline{m}}$ & $\hat{\sim} \stackrel{ \pm}{0}$ & 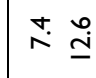 & 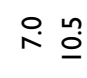 & 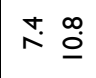 & 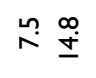 \\
\hline & 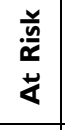 & 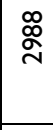 & 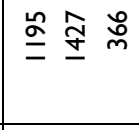 & $\stackrel{\circ}{\bar{\alpha}} \underset{\sim}{\stackrel{\circ}{\rho}}$ & $\overline{\bar{c}}$ 命 & $\underset{N}{\infty}$ & 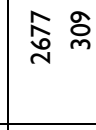 & $\hat{\sim} \bar{\sim}$ & 苟 & $\stackrel{\frac{N}{\infty} \stackrel{N}{N}}{=}$ \\
\hline \multirow{5}{*}{ 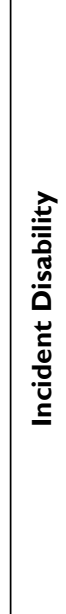 } & 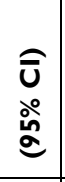 & & 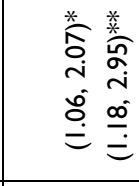 & 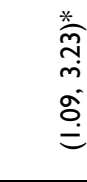 & 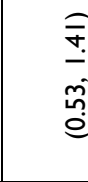 & $\begin{array}{l}\bar{\equiv} \\
\overline{0} \\
\stackrel{0}{0}\end{array}$ & $\begin{array}{l}\overline{0} \\
\stackrel{0}{0} \\
\stackrel{0}{0}\end{array}$ & 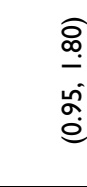 & 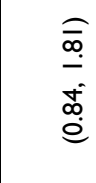 & 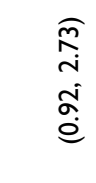 \\
\hline & 。̊ & & - $\stackrel{?}{.} \stackrel{\circ}{=}$ & $\stackrel{\infty}{-}$ & $\stackrel{\infty}{\infty}$ & $\stackrel{m}{=}$ & $\overline{\underline{a}}$ & $\stackrel{p}{9}$ & $\stackrel{\widetilde{T}}{=}$ & $\stackrel{\stackrel{\infty}{?}}{\rightarrow}$ \\
\hline & $\mathbf{z}$ & ब্d & 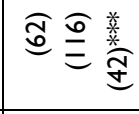 & 总 & 會 & 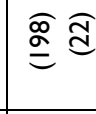 & 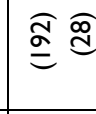 & 厓慈 & 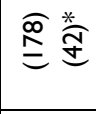 & 高产 \\
\hline & $\circ$ & $\infty$ & 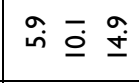 & $\stackrel{\sim}{\infty} \stackrel{\infty}{\stackrel{\infty}{\infty}}$ & 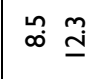 & $\stackrel{\circ}{\infty} \stackrel{\circ}{=}$ & $\infty \stackrel{\circ}{\stackrel{9}{=}}$ & $\hat{\sim} \stackrel{\infty}{\underline{I}}$ & $\stackrel{+}{\infty} \stackrel{a}{=}$ & $\stackrel{\circ}{\infty} \stackrel{\underline{m}}{\infty}$ \\
\hline & 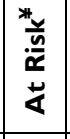 & 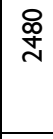 & 㙑 员 & $\overline{\mathrm{o}} \underset{\sim}{\mathrm{N}} \stackrel{\varrho}{=}$ & $\stackrel{\sim}{\mathcal{N}} \stackrel{\infty}{\stackrel{\infty}{\sim}}$ & 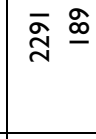 & 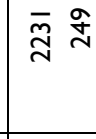 & $\begin{array}{l}\infty \\
\vdots \\
\varrho\end{array}$ & $\stackrel{\infty}{\stackrel{\infty}{N} \stackrel{\sim}{~} \tilde{m}}$ & 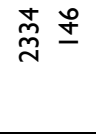 \\
\hline \multirow{5}{*}{ 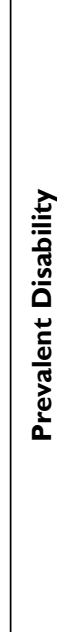 } & 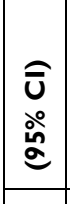 & & 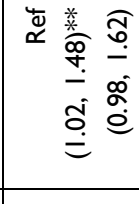 & 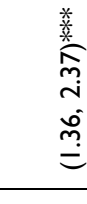 & 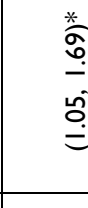 & 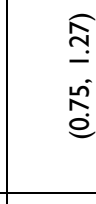 & פ & 稊 & 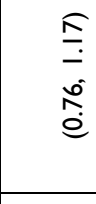 & 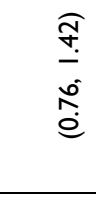 \\
\hline & $\stackrel{\circ}{0}$ & & 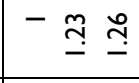 & $-\stackrel{\overbrace{}}{-}$ & $\stackrel{m}{=}$ & o. & $\stackrel{m}{=}$ & S. & ơ & $\stackrel{t}{-}$ \\
\hline & $\underline{\mathbf{z}}$ & 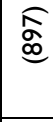 & 产全善言 & 善善 & 㷳蔨 & 㖟善 & 在善 & 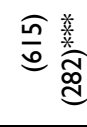 & 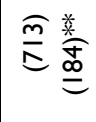 & $\underset{\Phi=\infty}{\widehat{Q}} \widehat{\Omega}$ \\
\hline & $\circ$ & $\underline{0}$ & $\stackrel{\circ}{=} \stackrel{\sim}{=} \underset{\sim}{\alpha}$ & 占 疋 & 实热 & $\underline{\tilde{0}} \tilde{\mathrm{N}}$ & $\underline{\sim} \stackrel{m}{i}$ & $\stackrel{m}{\underline{n}} \stackrel{.}{\bar{N}}$ & $\underline{\bar{\rho}} \stackrel{\infty}{\circ}$ & 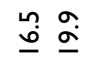 \\
\hline & 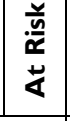 & 惫 & مू & 总 & 员品 & 总 & 芧 & 产产 & $\overline{\mathrm{g}}$ 合 & 总 $\bar{m}$ \\
\hline & & ○I & $\stackrel{0}{z} \stackrel{\tilde{u}}{\tau}$ & 욯 & $\stackrel{0}{z} \stackrel{0}{\check{0}}$ & 串 & $\stackrel{0}{2} \stackrel{0}{\sim}$ & 용 & 용 \\
\hline & & مَّم & 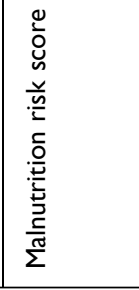 & 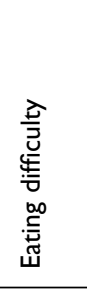 & 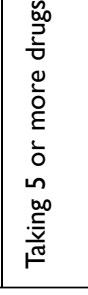 & 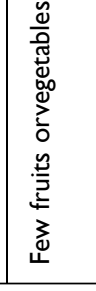 & 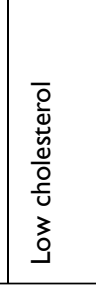 & 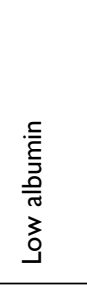 & 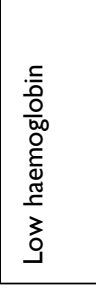 & 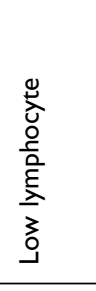 \\
\hline
\end{tabular}


ADL decline was observed in $7.9 \%$ of the participants overall, increasing from $5.3 \%$ to $8.3 \%$ to $14.8 \%$ across ordinal categories of MR. The OR adjusted for demographic, social, lifestyle behaviour and health variables was $1.26(95 \% \mathrm{CI}=0.90,1.76)$ for moderate $\mathrm{MR}$, and $1.65(95 \% \mathrm{CI}=1.08,2.53)$ for severe MR.

The risks of incident disability and ADL decline associated with specific indicators of MR are also shown in Table 2 and Supplementary Figure 1. The strongest associations that were robust to adjustment for demographic, social, lifestyle behaviour and health variables were for eating difficulty (chewing or swallowing) with incident disability $(\mathrm{OR}=1.88,95 \%$ CI $1.09,3.23)$ and functional decline $(\mathrm{OR}=1.63,95 \% \mathrm{CI}=1.01,2.64)$, and low lymphocyte with functional decline $(\mathrm{OR}=2.14,95 \%$ CI $1.33,3.44)$.

\section{Physical Function}

Among disability-free SLAS-2 participants, estimates of the levels and changes in KES, GV and TUG at baseline and follow-up by nutritional groups are shown in Supplementary Table 1 and Supplementary Figure 2. Significant nutritional group differences in the level and decline of physical performance after covariate adjustment were observed for overall high MR, consuming few vegetables or fruits, taking 5 or more drugs daily, low albumin, low cholesterol, and low haemoglobin. Compared to no MR, severe MR was associated with $50 \%$ worse decline in GV and doubly worse decline in TUG (Table 3 and Supplementary Figure 2). Consuming few vegetables or fruits was associated with $34 \%$ worse decline in KES, taking 5 or more drugs daily was associated with $56 \%$ worse TUG decline, and low albumin with $37 \%$ worse decline in GV and 91\% worse decline in TUG (Figure 2).

\section{Discussion}

In this prospective cohort study, we showed that community-dwelling older persons with malnutrition risk compared to their low risk peers were more likely to become disabled or show worsening disability. In addition, nondisabled older persons with malnutrition risk were more likely to show diminished or declining strength, gait and mobility over time, which are known to precede functional disability. Our study is among a few longitudinal studies that provide evidence in support of the influence of malnutrition risk in the disablement process leading to the development of functional decline and disability, ${ }^{22,23,26}$ and point to the potential impact of nutritional interventions for disability prevention in older people.
In the face of a global challenge of population ageing, there is wide consensus that ageing societies should strive to enable older people to remain in good health, and maintain their personal independence and activity involvement in the community. A cornerstone of ageing policy actions is the promotion and support of healthy ageing, and this should include the screening and treatment of malnutrition risk among non-disabled older people in the community. Recent studies provide strong support of the effectiveness of various lifestyle and health behaviour interventions in improving physical, cognitive and mood functioning of older adults, although evidence of ultimate effectiveness in preventing disability remain limited. Also, the evidence in support of the effectiveness of nutritional interventions compared to other interventions such as physical exercise or social activity interventions are relatively more limited. More studies involving nutritional interventions are required.

We used a highly appropriate measure of malnutrition risk for the purpose of our study. The Mini Nutritional Assessment (MNA) is most popularly used to measure nutritional risk and malnutrition. ${ }^{8-14}$ However, the MNA includes a question on immobility (being bed or chair bound) which is a component of ADL disability, and questions such as acute disease, dementia or depression, weight loss and BMI which are major risk factors for disability, hence there exists a circularity in this relationship. BMI has also been used to measure nutritional status in some studies; ${ }^{15-20}$ however, low BMI as a marker of muscle loss in malnutrition lacks sensitivity in older persons given the age-related increase in body weight, ${ }^{47}$ which may explain the reported lack of association with disability and physical function in many studies. Only a few studies which specifically measured low food intake and anorexia, ${ }^{21,22}$ low vegetable or fruit intake, ${ }^{23}$ or used blood biomarkers such as low albumin and low lymphocyte have reported positive findings. ${ }^{24-27}$

To minimize the bias in this study, we used a novel ENIGMA nutritional index which was previously shown to have good predictive validity for mortality risk. In this study, it is also demonstrated to predict disability. The ENIGMA assesses two major elements leading to malnutrition: undernutrition due to inadequate intake or digestion and intestinal absorption of food, and inflammation which leads to loss of fat free mass and negatively influences immune host response, healing and survival. ${ }^{47}$ Omitting inability to shop, cook or feed oneself (itself a measure of functional disability), the index includes 
Table 3 Adjusted Estimates of Between-Nutritional Group Differences in Decline in Physical Performance Among Disability-Free SLAS2 Participants

\begin{tabular}{|c|c|c|c|c|c|c|}
\hline & & \multirow{2}{*}{\multicolumn{2}{|c|}{$\begin{array}{c}\text { Decline from Baseline } \\
\text { Adjusted Mean }(95 \% \mathrm{Cl})\end{array}$}} & \multicolumn{3}{|c|}{ Between Group Differences } \\
\hline & & & & \multicolumn{2}{|c|}{ Adjusted Mean $(95 \% \mathrm{Cl})$} & $\%$ \\
\hline \multicolumn{7}{|l|}{ KES, kg } \\
\hline \multirow[t]{3}{*}{ Malnutrition risk score } & 0 & -2.63 & $(-3.44,-1.82)$ & 0 & Reference & 0 \\
\hline & $\mathrm{I}-2$ & -2.65 & $(-3.44,-1.86)$ & -0.02 & $(-0.55,0.5 I)$ & 0.8 \\
\hline & $3+$ & -2.46 & $(-3.38,-1.53)$ & 0.17 & $(-0.67,1.01)$ & 6.5 \\
\hline \multirow[t]{2}{*}{ Eating difficulty due to oral problems } & No & -2.58 & $(-3.31,-1.85)$ & 0 & & \\
\hline & Yes & -2.33 & $(-3.54,-1.13)$ & 0.24 & $(-0.75,1.24)$ & 9.3 \\
\hline \multirow[t]{2}{*}{ Taking 5 or more drugs } & No & -2.65 & $(-3.38,-0.19)$ & 0 & & \\
\hline & Yes & -2.52 & $(-0.35,-0.59)$ & 0.13 & $(-0.64,0.89)$ & 4.9 \\
\hline \multirow[t]{2}{*}{ Few fruits or vegetables } & No & -2.70 & $(-3.44,-1.96)^{* * *}$ & 0 & & \\
\hline & Yes & -1.76 & $(-2.8 \mathrm{I},-0.7 \mathrm{I})^{* *}$ & 0.94 & $(0.07,1.82)^{*}$ & 34.8 \\
\hline \multirow[t]{2}{*}{ Low cholesterol } & No & -2.58 & $(-3.33,-1.82)$ & 0 & & \\
\hline & Yes & -2.73 & $(-3.65,-1.81)$ & -0.16 & $(-0.89,0.57)$ & 6.2 \\
\hline \multirow[t]{2}{*}{ Low albumin } & No & -2.50 & $(-3.25,-1.74)$ & 0 & & \\
\hline & Yes & -2.81 & $(-3.68,-1.93)$ & -0.31 & $(-0.93,0.31)$ & 11.3 \\
\hline \multirow[t]{2}{*}{ Low haemoglobin } & No & -2.55 & $(-3.31,-1.80)$ & 0 & & \\
\hline & Yes & -2.79 & $(-3.68,-1.90)$ & -0.23 & $(-0.87,0.41)$ & 9.0 \\
\hline \multirow[t]{2}{*}{ Low lymphocyte count } & No & -2.60 & $(-3.34,-1.86)$ & 0 & & \\
\hline & Yes & -2.66 & $(-3.87,-1.45)$ & -0.06 & $(-1.04,0.91)$ & 2.3 \\
\hline \multicolumn{7}{|l|}{$\mathbf{G V}, \mathbf{m} / \mathbf{s}$} \\
\hline \multirow[t]{3}{*}{ Malnutrition risk score } & 0 & -0.14 & $(-0.20,-0.09)$ & 0 & Reference & 0 \\
\hline & $\mathrm{I}-2$ & -0.17 & $(-0.23,-0.12)^{*}$ & -0.03 & $(-0.07,-0.01)^{*}$ & 21.4 \\
\hline & $3+$ & -0.21 & $(-0.28,-0.15)^{*}$ & -0.07 & $(-0.13,-0.001)^{*}$ & 50.0 \\
\hline \multirow[t]{2}{*}{ Eating difficulty due to oral problems } & No & -0.16 & $(-0.21,-0.1 \mathrm{I})$ & 0 & & \\
\hline & Yes & -0.20 & $(-0.28,-0.12)$ & -0.04 & $(-0.11,0.03)$ & 25.0 \\
\hline \multirow[t]{2}{*}{ Taking 5 or more drugs } & No & -0.16 & $(-0.22,-0.11)$ & 0 & & \\
\hline & Yes & -0.22 & $(-0.27,-0.15)$ & -0.05 & $(-0.10,0.01)$ & 31.2 \\
\hline \multirow[t]{2}{*}{ Few fruits or vegetables } & No & -0.16 & $(-0.21,-0.11)$ & 0 & & \\
\hline & Yes & -0.20 & $(-0.27,-0.12)$ & -0.04 & $(-0.10,0.03)$ & 25.0 \\
\hline \multirow[t]{2}{*}{ Low cholesterol } & No & -0.16 & $(-0.21,-0.11)$ & 0 & & \\
\hline & Yes & -0.20 & $(-0.27,-0.13)$ & -0.04 & $(-0.09,0.01)$ & 25.0 \\
\hline \multirow[t]{2}{*}{ Low albumin } & No & -0.16 & $(-0.21,-0.10)$ & 0 & & \\
\hline & Yes & -0.22 & $(-0.28,-0.15)^{* *}$ & -0.06 & $(-0.10,-0.02)^{* *}$ & 37.5 \\
\hline \multirow[t]{2}{*}{ Low haemoglobin } & No & -0.17 & $(-0.22,-0.12)$ & 0 & & \\
\hline & Yes & -0.18 & $(-0.24,-0.12)$ & -0.01 & $(-0.06,0.03)$ & 5.9 \\
\hline \multirow[t]{2}{*}{ Low lymphocyte count } & No & -0.17 & $(-0.22,-0.12)$ & 0 & & \\
\hline & Yes & -0.20 & $(-0.28,-0.11)$ & -0.03 & $(-0.09,0.04)$ & 17.6 \\
\hline \multicolumn{7}{|l|}{ TUG, sec } \\
\hline \multirow[t]{3}{*}{ Malnutrition risk score } & 0 & 0.61 & $(0.10,1.12)$ & 0 & Reference & 0 \\
\hline & $\mathrm{I}-2$ & 0.96 & $(0.46,1.46)$ & 0.34 & $(-0.03,0.72)$ & 55.7 \\
\hline & $3+$ & 1.38 & $(0.79,1.97)^{* *}$ & 0.77 & $(0.17,1.37)^{* *}$ & 126.2 \\
\hline
\end{tabular}

(Continued) 
Table 3 (Continued).

\begin{tabular}{|c|c|c|c|c|c|c|}
\hline \multirow{4}{*}{ Eating difficulty due to oral problems } & \multirow{4}{*}{$\begin{array}{l}\text { No } \\
\text { Yes }\end{array}$} & \multirow{2}{*}{\multicolumn{2}{|c|}{$\begin{array}{l}\text { Decline from Baseline } \\
\text { Adjusted Mean }(95 \% \mathrm{Cl})\end{array}$}} & \multicolumn{3}{|c|}{ Between Group Differences } \\
\hline & & & & \multicolumn{2}{|c|}{ Adjusted Mean (95\% Cl) } & \multirow{3}{*}{$\begin{array}{r}\% \\
57.4\end{array}$} \\
\hline & & 1.01 & $(0.54,1.48)$ & 0 & & \\
\hline & & 1.59 & $(0.83,2.35)$ & 0.58 & $(-0.05,1.21)$ & \\
\hline \multirow[t]{2}{*}{ Taking 5 or more drugs } & No & 1.05 & $(0.59,1.52)$ & 0 & & \\
\hline & Yes & 1.65 & $(1.04,2.26)^{*}$ & 0.59 & $(0.10,1.09)^{*}$ & 56.2 \\
\hline \multirow[t]{2}{*}{ Few fruits or vegetables } & No & 1.04 & $(0.57,1.52)$ & 0 & & \\
\hline & Yes & 1.03 & $(0.35,1.72)$ & -0.007 & $(-0.580,0.565)$ & 0.7 \\
\hline \multirow[t]{2}{*}{ Low cholesterol } & No & 0.85 & $(0.367,1.33)$ & 0 & & \\
\hline & Yes & 1.14 & $(0.55,1.74)$ & 0.29 & $(-0.18,0.76)$ & 34.1 \\
\hline \multirow{2}{*}{ Low albumin } & No & 0.77 & $(0.29,1.24)$ & 0 & & \\
\hline & Yes & 1.46 & $(0.90,2.02)^{* * *}$ & 0.70 & $(0.30,1.08)^{* * *}$ & 90.9 \\
\hline \multirow[t]{2}{*}{ Low haemoglobin } & No & 0.91 & $(0.43,1.39)$ & 0 & & \\
\hline & Yes & 0.99 & $(0.43,1.56)$ & 0.09 & $(-0.32,0.49)$ & 9.9 \\
\hline \multirow[t]{2}{*}{ Low lymphocyte count } & No & 0.91 & $(0.44,1.38)$ & 0 & & \\
\hline & Yes & 1.40 & $(0.65,2.16)$ & 0.49 & $(-0.12,1.11)$ & 53.8 \\
\hline
\end{tabular}

Notes: Ref: Reference; ${ }^{*} p<0.05,{ }^{* *} p<0.01,{ }^{* * *} p<0.001$ (Bonferroni-adjusted). Adjusted for age, sex, ethnicity, education, housing type, marital status, living arrangement, hours of moderate to high physical activity, number of comorbidities, GDS, MMSE and baseline KES (or GV or TUG). Deterioration from baseline is measured with appropriate reverse signs for changes from baseline of KES, GV and TUG.

indicators of reduced quantity and quality of food intake due to difficulty in chewing and swallowing, and low albumin, cholesterol, haemoglobin and lymphocyte count which assess negative nitrogen balance, inflammatory activity and impaired immune host response. Furthermore, as body weight tends to increase with age, and low BMI and weight loss in older people are less able to detect the crucial loss of lean muscle mass associated with undernutrition. ${ }^{47} \mathrm{We}$ and others have previously observed that BMI in the presence of other nutritional variables was a redundant in predicting mortality risk. ${ }^{28,48}$ Furthermore, BMI was also not found in previous studies to be consistently associated with risk of disability or impaired physical performance. ${ }^{15-20}$

Eating difficulty alone was found to be most strongly associated with increased risk of incident disability and functional decline in ADL. This result is congruent with two previous prospective studies demonstrating that anorexia of ageing (loss of appetite or reduced food intake) was associated with risk of incident disability. ${ }^{21,22}$ Our study also identified low lymphocyte count as a malnutrition indicator that predicted functional $\mathrm{ADL}$ decline. Low lymphocyte count has also been reported to be associated with ADL disability in a study of Chinese centenarian women. ${ }^{27}$
With regard to physical function, we also showed that malnutrition risk and low albumin in particular were associated with worse decline in GV and TUG, consistent with previously reported associations of low serum albumin with low muscle mass ${ }^{25}$ and ADL limitations. ${ }^{26}$ Longterm protein-energy malnutrition and inflammation from disease and injury are principally responsible for low serum albumin concentrations in older persons, and its associated risk of physical function loss and disability is likely to be attributable to sarcopenia. ${ }^{24}$ Polypharmacy is widely regarded to be an important risk factor for malnutrition based on its documented effects on alterations in taste, common gastrointestinal side effects such as nausea, anorexia and dry mouth, or intestinal absorption and metabolism or elimination of vitamins and minerals. ${ }^{49}$ It is also regarded as an important risk factor for frailty and disability risk, although a systematic review reported that the data, based on cross-sectional studies, are mixed regarding the relationship between polypharmacy and a range of adverse outcomes including function. ${ }^{50}$ More recently, however, several longitudinal studies have emerged in support of this association with incident frailty. ${ }^{51-53}$

Consuming few vegetables or fruits (less than once a day) was significantly associated with worse decline in knee extension strength in this study, in agreement with 

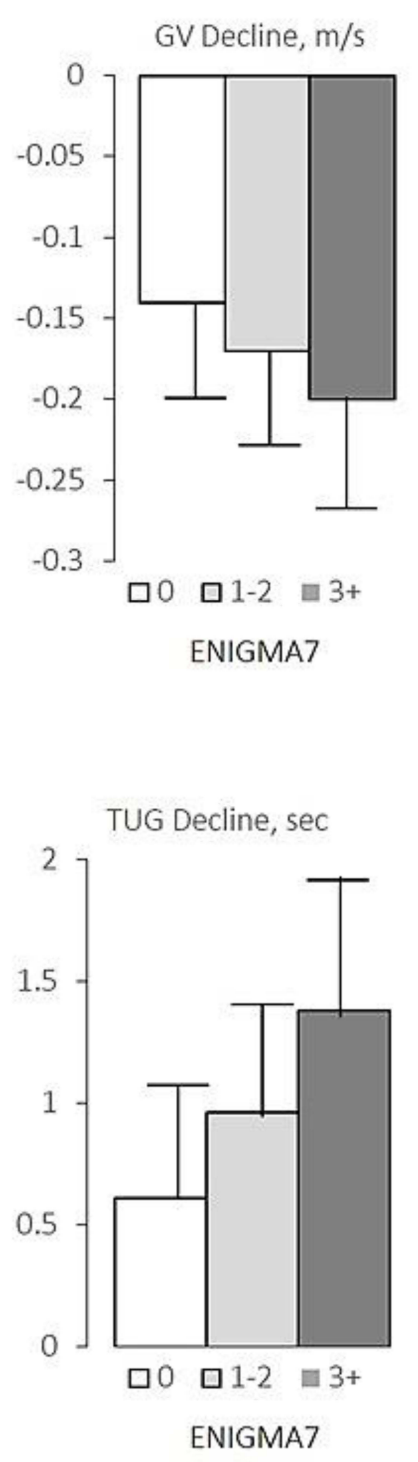
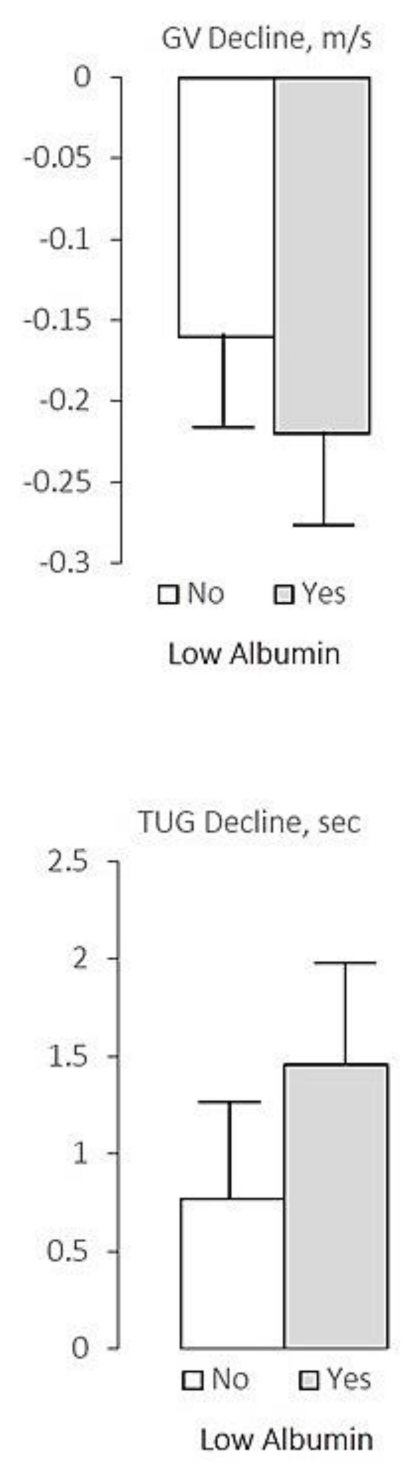

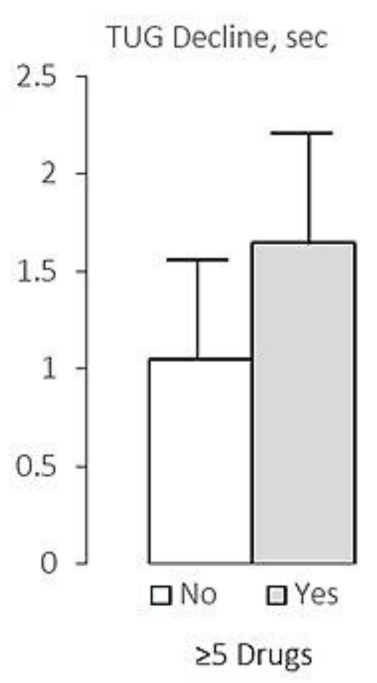

Figure 2 Significant estimates of GV and TUG declines at follow-up associated with ENIGMA7 malnutrition risk, low albumin and consumption of $\geq 5$ drugs among disabilityfree SLAS-2 participants.

the finding reported in one previous longitudinal study of African-Americans. $^{23}$ This is consonant with studies which suggested the associations of single nutrient dietary intake, such as carotenoids, vitamins B, D, vitamins B and folate, selenium, and the Mediterranean diet with impaired physical function or frailty. ${ }^{7}$

\section{Strengths and Limitations}

This study used a large prospective cohort of communitydwelling middle-aged and older persons and a careful and appropriate choice of instrument to measure nutrition. However, some limitations should be noted. The relatively smaller number of old-old individuals in this cohort may limit generalizing the findings to much older people, among whom some studies suggest the association of nutritional factors with physical functional decline may be attenuated or even paradoxical. ${ }^{18}$ Given the 3 to 5 -year interval between assessment of physical function and decline, it is possible that transitions over short intervals may be missed. Some degree of selection bias should be noted as the study cohort excluded very disabled older persons in the community, and more physically impaired, frail or disabled participants among them were lost to follow-up. Attrition bias should also be considered as participants who were lost to follow-up or died had more malnutrition risk and were more disabled at baseline than those who were assessed at follow-up. This was likely to result in the risks of disability and levels of physical and 
functional performance associated with malnutrition risk being underestimated. Specific nutritional factors such as dietary intake or biochemical levels of specific nutrients or dietary patterns are not assessed in this study.

\section{Conclusion}

In summary, this study showed that malnutrition risk increased the risk of incident disability and worsening disability, and worse declines in physical function among non-disabled individuals. The contributing factors included reduced food intake because of chewing or swallowing difficulty, few intakes of vegetables or fruits, polypharmacy, low albumin, and low lymphocyte count. Further studies especially interventional studies should be conducted to guide preventive efforts for reducing disability among older people.

\section{Acknowledgments}

We thank the following voluntary welfare organizations for their support: Geylang East Home for the Aged, Presbyterian Community Services, Thye Hua Kwan Moral Society (Moral Neighbourhood Links), Yuhua Neighbourhood Link, Henderson Senior Citizen's Home, National Trade Union Congress Eldercare Co-op Ltd, Thong Kheng Senior Activity Centre (Queenstown) and Redhill Moral Seniors Activity Centre.

\section{Disclosure}

Professor Tze Pin Ng report grants from Agency for Science, Technology and Research (A*STAR), grants from Ministry of Health (MOH) National Medical Research Council, during the conduct of the study. The authors report no other conflicts of interest in this work.

\section{References}

1. Micha R, Mannar V, Afshin A, et al. 2020 Global Nutrition Report: Action on Equity to End Malnutrition. Bristol, UK: Development Initiatives; 2020.

2. Cereda E, Pedrolli C, Klersy C, et al. Nutritional status in older persons according to healthcare setting: a systematic review and metaanalysis of prevalence data using MNA $^{\circledR}$. Clin Nutr. 2016;35 (6):1282-1290. doi:10.1016/j.clnu.2016.03.008

3. Verlaan S, Ligthart-Melis GC, Wijers SLJ, et al. High prevalence of physical frailty among community-dwelling malnourished older adults-a systematic review and meta-analysis. $\mathrm{J} \mathrm{Am} \mathrm{Med} \mathrm{Dir} \mathrm{Assoc.}$ 2017;18(5):374-382. doi:10.1016/j.jamda.2016.12.074

4. Chapman IM. Nutritional disorders in the elderly. Med Clin North Am. 2006;90(5):887-907. doi:10.1016/j.mcna.2006.05.010

5. World Health Organization. The Global Strategy and Action Plan on Ageing and Health (2016-2020). World Health Organization; 2016.
6. Fried TR, Bradley EH, Williams CS, et al. Functional disability and health care expenditures for older persons. Arch Intern Med. 2001;161(21):2602-2607. doi:10.1001/archinte.161.21.2602

7. Cederholm T, Nouvenne A, Ticinesi A, et al. The role of malnutrition in older persons with mobility limitations. Curr Pharm Des. 2014;20 (19):3173-3177. doi:10.2174/13816128113196660689

8. Tramontano A, Veronese N, Giantin V, et al. Nutritional status, physical performance and disability in the elderly of the Peruvian Andes. Aging Clin Exp Res. 2016;28(6):1195-1201. doi:10.1007/ s40520-016-0591-9

9. Corona LP, Pereira de Brito TR, Nunes DP, et al. Nutritional status and risk for disability in instrumental activities of daily living in older Brazilians. Public Health Nutr. 2014;17(2):390-395. doi:10.1017/ S1368980012005319

10. Danielewicz AL, Barbosa AR, Del Duca GF. Nutritional status, physical performance and functional capacity in an elderly population in southern Brazil. Rev Assoc Med Bras. 2014;60(3):242-248. doi:10.1590/1806-9282.60.03.0013

11. Ramsey KA, Meskers CGM, Trappenburg MC, et al. Malnutrition is associated with dynamic physical performance. Aging Clin Exp Res. 2020;32(6):1085-1092. doi:10.1007/s40520-019-01295-3

12. Martínez-Reig M, Gómez-Arnedo L, Alfonso-Silguero SA, et al. Nutritional risk, nutritional status and incident disability in older adults. The FRADEA study. $J$ Nutr Health Aging. 2014;18 (3):270-276. doi:10.1007/s12603-013-0388-x

13. Hsu YH, Chou MY, Chu CS, et al. Predictive effect of malnutrition on long-term clinical outcomes among older men: a prospectively observational cohort study. $J$ Nutr Health Aging. 2019;23 (9):876-882. doi:10.1007/s12603-019-1246-2

14. Misu S, Asai T, Doi T, et al. Association between gait abnormality and malnutrition in a community-dwelling elderly population. Geriatr Gerontol Int. 2017;17(8):1155-1160. doi:10.1111/ggi.12839

15. Nam S, Kuo YF, Markides KS, et al. Waist circumference (WC), body mass index (BMI), and disability among older adults in Latin American and the Caribbean (LAC). Arch Gerontol Geriatr. 2012;55 (2):e40-e47. doi:10.1016/j.archger.2012.04.006

16. Ramsay SE, Whincup PH, Shaper AG, et al. The relations of body composition and adiposity measures to ill health and physical disability in elderly men. Am $J$ Epidemiol. 2006;164(5):459-469. doi:10.1093/aje/kwj217

17. Lv YB, Yuan JQ, Mao C, et al. Association of body mass index with disability in activities of daily living among Chinese adults 80 years of age or older. JAMA Netw Open. 2018;1(5):e181915. doi:10.1001/ jamanetworkopen.2018.1915

18. Lisko I, Tiainen K, Raitanen J, et al. Body mass index and waist circumference as predictors of disability in nonagenarians: the Vitality 90+ Study. J Gerontol A Biol Sci Med Sci. 2017;72 (11):1569-1574. doi:10.1093/gerona/glx032

19. Sergi G, Perissinotto E, Toffanello ED, et al. Lower extremity motor performance and body mass index in elderly people: the Italian Longitudinal Study on Aging (ILSA). J Gerontol a Biol Sci Med Sci. 2007;55(12):2023-2029.

20. Ferreira RS, da Silva Coqueiro R, Barbosa AR, et al. Relationship between BMI and physical performance among older adults. Geriatr Nurs. 2013;34(6):465-468. doi:10.1016/j.gerinurse.2013.07.013

21. Tsutsumimoto K, Doi T, Makizako H, et al. Aging-related anorexia and its association with disability and frailty. $J$ Cachexia Sarcopenia Muscle. 2018;9(5):834-843. doi:10.1002/jcsm.12330

22. Landi F, Russo A, Liperoti R, et al. Anorexia, physical function, and incident disability among the frail elderly population: results from the ilSIRENTE study. J Am Med Dir Assoc. 2010;11(4):268-274. doi:10.1016/j.jamda.2009.12.088

23. Ribeiro SM, Morley JE, Malmstrom TK, et al. Fruit and vegetable intake and physical activity as predictors of disability risk factors in African-American middle-aged individuals. $J$ Nutr Health Aging. 2016;20(9):891-896. doi:10.1007/s12603-016-0780-4 
24. Salive ME, Cornoni-Huntley J, Phillips CL, et al. Serum albumin in older persons: relationship with age and health status. J Clin Epidemiol. 1992;45(3):213-221. doi:10.1016/0895-4356(92)90081-W

25. Baumgartner RN, Koehler KM, Romero L, et al. Serum albumin is associated with skeletal muscle in elderly men and women. Am J Clin Nutr. 1996;64(4):552-558. doi:10.1093/ajcn/64.4.552

26. Uemura K, Doi T, Lee S, Shimada H. Sarcopenia and low serum albumin level synergistically increase the risk of incident disability in older adults. J Am Med Dir Assoc. 2019;20(1):90-93. doi:10.1016/j. jamda.2018.06.011

27. Liu Z, Wang Y, Huang J, et al. Blood biomarkers and functional disability among extremely longevous individuals: a population-based study. J Gerontol a Biol Sci Med Sci. 2015;70 (5):623-627. doi:10.1093/gerona/glu229

28. Ng TP, Nyunt MSZ, Gao Q, et al. Elderly Nutritional Indicators for Geriatric Malnutrition Assessment (ENIGMA): development and validation of a nutritional prognostic index. Clin Nutr ESPEN. 2017;22:54-63. doi:10.1016/j.clnesp.2017.08.012

29. Ng TP, Niti M, Feng L, Kua EH, Yap KB. Albumin, apolipoprotein E-epsilon4 and cognitive decline in community-dwelling Chinese older adults. J Am Geriatr Soc. 2009;57(1):101-106. doi:10.1111/ j.1532-5415.2008.02086.x

30. Ng TP, Feng L, Nyunt MSZ, Larbi A, Yap KB. Frailty in older persons: multisystem risk factors and the Frailty Risk Index (FRI). J Am Med Dir Assoc. 2014;15(9):635-642. doi:10.1016/j.jamda.2014.03.008

31. Posner BM, Jette AM, Smith KW, Miller DR. Nutrition and health risks in the elderly: the nutrition screening initiative. Am J Public Health. 1993;83(7):972-978. doi:10.2105/AJPH.83.7.972

32. Rubenstein LZ, Harker JO, Salva A, Guigoz Y, Vellas B. Screening for undernutrition in geriatric practice: developing the short-form Mini Nutritional Assessment (MNA-SF). J Gerontol. 2001;56(6): M366-M372. doi:10.1093/gerona/56.6.M366

33. Penninx BW, Pahor M, Woodman RC, Guralnik JM. Anemia in old age is associated with increased mortality and hospitalization. J Gerontol a Biol Sci Med Sci. 2006;61(5):474-479. doi:10.1093/gerona/61.5.474

34. Chalmers KA, Knuiman MW, Divitini ML, Bruce DG, Olynyk JK, Milward EA. Long-term mortality risks associated with mild anaemia in older persons: the Busselton Health Study. Age Ageing. 2012;41 (6):759-764. doi:10.1093/ageing/afs150

35. Rubio-Rivas M, Formiga F, Grillo S, Gili F, Cabrera C, Corbella X. Lymphopenia as prognostic factor for mortality and hospital length of stay for elderly hospitalized patients. Aging Clin Exp Res. 2016;28 (4):721-727. doi:10.1007/s40520-015-0474-5

36. Okamura T, Hayakawa T, Hozawa A, et al. Lower levels of serum albumin and total cholesterol associated with decline in activities of daily living and excess mortality in a 12-year cohort study of elderly Japanese. J Am Geriatr Soc. 2008;56(3):529-535. doi:10.1111/ j.1532-5415.2007.01549.x

37. Volpato S, Leveille SG, Corti MC, Harris TB, Guralnik JM. The value of serum albumin and high-density lipoprotein cholesterol in defining mortality risk in older persons with low serum cholesterol. J Am Geriatr Soc. 2001;49(9):1142-1147. doi:10.1046/j.1532-5415.2001.49229.x

38. Reuben DB, Ix JH, Greendale GA, Seeman TE. The predictive value of combined hypoalbuminemia and hypocholesterolemia in high functioning community-dwelling older persons: MacArthur studies of successful aging. J Am Geriatr Soc. 1999;47(4):402-406. doi:10.1111/j.1532-5415.1999.tb07230.x
39. Ng TP, Niti M, Chiam PC, Kua EH. Prevalence and correlates of functional disability in multiethnic elderly Singaporeans. $J$ Am Ger Soc. 2006;54(1):21-29. doi:10.1111/j.1532-5415.2005.00533.x

40. Niti M, Chiam PC, Kua EH, Ng TP. Physical and cognitive domains of the Instrumental Activities of Daily Living: validation in a multiethnic population of Asian older adults. $J$ Gerontol a Biol Sci Med Sci. 2006;61(7):726-735. doi:10.1093/gerona/61.7.726

41. Spector WD, Katz S, Murphy JB, Fulton JP. The hierarchical relationship between activities of daily living and instrumental activities of daily living. J Chron Dis. 1987;40(6):481-489. doi:10.1016/0021-9681(87) 90004-X

42. LaPlante MP. The classic measure of disability in activities of daily living is biased by age but an expanded IADL/ADL measure is not. $J$ Gerontol B Psychol Sci Soc Sci. 2010;65B(6):720-732. doi:10.1093/geronb/gbp129

43. Lord SR, Menz HB, Tiedemann A. A physiological profile approach to falls risk assessment and prevention. Phys Ther. 2003;83 (3):237-252. doi:10.1093/ptj/83.3.237

44. Choo PL, Tou NX, Jun Pang BW, et al. Timed Up and Go (TUG) reference values and predictive cutoffs for fall risk and disability in Singaporean community-dwelling adults: Yishun cross-sectional study and Singapore longitudinal aging study. J Am Med Dir Assoc. 2021;22 (8):1640-1645. doi:10.1016/j.jamda.2021.03.002

45. Ng TP, Niti M, Chiam PC, Kua EH. Ethnic differences in cognitive performance on mini-mental state examination in Asians. Am J Geriatr Psychiatry. 2007;15(2):130-139. doi:10.1097/01.JGP.0000 235710.17450.9a

46. Nyunt MSZ, Jin AZ, Fones CSL, Ng TP. Criterion-based validity and reliability of the Geriatric Depression Screening Scale (GDS-15) in a large validation sample of community-living Asian older adults. Ageing Ment Health. 2009;13(3):376-382. doi:10.1080/13607860902861027

47. Soeters P, Bozzetti F, Cynober L, et al. Defining malnutrition: a plea to rethink. Clin Nutr. 2017;36(3):896-901. doi:10.1016/j.clnu.2016.09.032

48. Buys DR, Roth DL, Ritchie CS, et al. Nutritional risk and body mass index predict hospitalization, nursing home admissions, and mortality in community-dwelling older adults: results from the UAB study of aging with 8.5 years of follow-up. J Gerontol a Biol Sci Med Sci. 2014;69(9):1146-1153. doi:10.1093/gerona/glu024

49. Little MO. Updates in nutrition and polypharmacy. Curr Opin Clin Nutr Metab Care. 2018;21(1):4-9. doi:10.1097/MCO.0000000000000425

50. Favaro-Moreira NC, Krausch-Hofmann S, Matthys C, et al. Risk factors for malnutrition in older adults: a systematic review of the literature based on longitudinal data. Adv Nutr. 2016;7(3):507-522. doi:10.3945/an.115.011254

51. Wang R, Chen L, Fan L, et al. Incidence and effects of polypharmacy on clinical outcome among patients aged 80p: a five-year follow-up study. PLoS One. 2015;10(11):e0142123. doi:10.1371/journal.pone.0142123

52. Saum KU, Schottker B, Meid AD, et al. Is polypharmacy associated with frailty in older people? Results from the ESTHER cohort study. J Am Geriatr Soc. 2017;65(2):e27e32. doi:10.1111/jgs.14718

53. Herr M, Cesari M, Landre B, et al. Factors associated with changes of the frailty status after age 70: findings in the MAPT study. Ann Epidemiol. 2019;34:65-70.e1. doi:10.1016/j.annepidem.2019.03.008 


\section{Publish your work in this journal}

Clinical Interventions in Aging is an international, peer-reviewed journal focusing on evidence-based reports on the value or lack thereof of treatments intended to prevent or delay the onset of maladaptive correlates of aging in human beings. This journal is indexed on PubMed Central, MedLine, CAS, Scopus and the Elsevier
Bibliographic databases. The manuscript management system is completely online and includes a very quick and fair peer-review system, which is all easy to use. Visit http://www.dovepress.com/ testimonials.php to read real quotes from published authors.

Submit your manuscript here: https://www.dovepress.com/clinical-interventions-in-aging-journal 